\begin{tabular}{|c|c|}
\hline Title & $\begin{array}{l}\text { Performance A naly sis of Thermoelectric Modules Consisting of Square Truncated Pyramid Elements Under Constant } \\
\text { Heat Flux }\end{array}$ \\
\hline Author(s) & Oki, Sae; Natsui, Shungo; Suzuki, Ryosuke O. \\
\hline Citation & $\begin{array}{l}\text { Journal of electronic materials, } 47(6), 3288-3297 \\
\text { https://doi.org/10.1007/s11664 017-6055-y }\end{array}$ \\
\hline Issue Date & 201806 \\
\hline Doc URL & http:/hdl .handle.net/2115/74513 \\
\hline Rights & $\begin{array}{l}\text { This is a post-peer-review, pre copyedit version of an article published in [Journal of electronic materials]. The final } \\
\text { authenticated version is avail able online at: } \mathrm{http}: / / \mathrm{dx} \text {.doi.org/10.1007/s11664 017-6055-y] }\end{array}$ \\
\hline Type & article (author version) \\
\hline File Information & ICT 20170ki_Ry020_White-1.pdf \\
\hline
\end{tabular}

Instructions for use 


\title{
Performance Analysis of Thermoelectric Modules Consisting of Square Truncated Pyramid Elements Under Constant Heat Flux
}

4

\section{Abstract}

1

to improve the TE performance. Square truncated pyramid shaped $\mathrm{P}-\mathrm{N}$ pairs of TE elements are connected electronically in series in the open space between two flat insulator boards. The performance of the TE module consisting of 2-paired elements is numerically simulated using commercial software and original TE programs.

\author{
Sae Oki*, Shungo Natsui and Ryosuke O. Suzuki**
}

Faculty of Engineering, Hokkaido University, Kita-13 Jou, Nishi-8 Chome, Kita-Ku, Sapporo, Hokkaido, 060-8628 Japan

*s_oki@eng.hokudai.ac.jp, ** Contributing author: rsuzuki@eng.hokudai.ac.jp

System design of thermoelectric (TE) power generation module is pursued in order Assuming that the heat radiating into the hot surface is regulated, i.e., the amount of heat from the hot surface to the cold one is steadily constant, as it happens for solar radiation heating, the performance is significantly improved by changing the shape and the alignment pattern of the elements. When the angle $\theta$ between the edge and the base is smaller than $72^{\circ}$, and when the cold surface is kept at a constant temperature, two patterns in particular, amongst the 17 studied, show the largest TE power and efficiency. In comparison to other geometries, the smarter square truncated pyramid shape can provide higher performances using a large cold bath and constant heat transfer by heat radiation. 


\section{Keywords:}

27 Thermoelectric power generation, constant heat flux, optimum element shape,

28 numerical simulation, square truncated pyramid element 


\section{Introduction}

Shapes of thermoelectric (TE) elements ${ }^{(1-8)}$ and interesting structures of TE modules (TEM) ${ }^{(9-12)}$ based on heat flow analysis have been recently proposed. The traditional П-type TE elements are sandwiched between two flat substrates with a heat supplier and a heat receiver, and the thermal heat, introduced from the higher temperature side, transfers to the lower temperature side through TE elements. Some portions of this transferring heat are converted to electricity in the TE material. In addition to the improvement of the performances of the materials, the optimized TE elements design techniques, using three-dimensional simulations along with simultaneous derivative equations, ${ }^{(4-8)}$ predict a larger conversion efficiency for the TEM. Varying its length, a segmented TE element was optimized for the best performance ${ }^{(1)}$. Other shapes such as cylindrical ${ }^{(2,3)}$, parallelogram-like ${ }^{(4-6)}$, trapezoid-like ${ }^{(7)}$, truncated square pyramid-like shapes ${ }^{(8)}$ have been analyzed. These studies optimized the shapes of TE elements by setting a certain constant temperature difference between the two substrates ${ }^{(1-8)}$. The assumption of constant temperature difference is quite easy and suitable for explicit mathematical solutions; however, constant temperature assumptions have a severe practical discrepancy because the majority of TE modules do not work in such an ideal setting; an infinite large thermal bath is required to insure a certain constant fixed temperature, although the assumption of constant temperature difference has been used diffusely since the TE phenomenon was found.

On the other hand, a steady heat flow from the hot side may be practically available especially in case of heat radiation. For example, solar heat radiation is in excellent approximation a constant heat source that determines the temperature difference in a TE 
54 module and allows a constant heat flow ${ }^{(13,14)}$. Depending on the solar energy, the hot side temperature varies allowing TE power generation. Radiation heat from a hot steel slab just after continuous casting is another good example that has been studied lately in Japanese industries ${ }^{(15)}$.

This study examines a design suitable for TE power generation utilizing heat radiation, where the conventional two flat substrates are taken as the basic structure of the TEM. The flat TE panel is commonly used to catch and convert the radiation heat, and a performance enhancement is expected with the introduction of a TE design. The TE analysis presented here is based on a mathematical approach.

Our previous work proposed the square truncated pyramidal shape (Fig.1) as a possible geometry for TE elements ${ }^{(8)}$, and a slightly slant structure gave both better performance and better efficiency than the conventional П-type TEM. This shape can suppress the heat transfer from the hot side to the colder side, for instance, when the smaller square surface of a TE element faces the hot surface (Fig. 2). Thanks to the small cross-sectional area of the TE element near the hot side, the heat penetrating into the TE elements may be cut off, and the hot side can be kept at higher temperature.

The purpose of this work is to optimize the shape of TE elements theoretically by taking into account that maximum TE power can be obtained with square truncated pyramids while in conditions of constant heat flux. As shown in Fig. 1, the TE performances are measured as a function of the angle between a base and an edge, $\theta$. This shape has four side faces, which may be subject to environmental effects due to the other surrounding elements (such as mutual radiation). In some cases, radiation among TE elements cannot be neglected, and this interaction may contribute constructively to the overall TE output performance. Therefore, 2 pairs of TE elements will be taken as 
fundamental building block for the analysis of TEM design (Fig. 3).

Our previous analysis using parallelepiped ${ }^{(4-6)}$ and square truncated pyramids ${ }^{(8)}$ assumed that the temperatures on both the hot and cold surfaces are constant. The performance was maximized at an angle, $\theta$, close to 85 degrees by changing the element shape. The alignment pattern of the elements in the TEM also affected performance and efficiency ${ }^{(8,9,12)}$. This work is based on the mathematical procedure developed and used in previous works ${ }^{(16,17)}$, but the requisite for external environment is adjusted for radiation heating.

\section{Model description and calculation conditions}

The length of the basal edge of the cubic TE element is set constant and equal to 1 mm. In order to be able to compare the results with those of the previous work ${ }^{(8)}$, the total volumes of a p- and n-type TE element is set to be $1 \mathrm{~mm}^{3}$, as shown in Fig. 4 . This assumption is conceived to find a better performance using the same amount of TE material. The angle between the base and the edge, $\theta$, is variable, and the height of the element, $h$, depends from $\theta$. $h$ becomes bigger as $\theta$ is reduced. The angular dependency of $h$ can be fitted with a parabolic approximation. The other variables of the TE element, such as b and c (in Fig. 4), are a function of $a$ and $\theta$, when $V$ is kept constant.

Four TE elements are electrically connected in series by thin electrodes, forming a TEM with two flat insulating plates and 5 electrodes, as shown in Fig. 3. $\theta$ is varied in the range from $72^{\circ}$ to $90^{\circ}$. For $\theta=90^{\circ}$, this module is equal to the conventional $\Pi$-type module with cubic TE elements. The ratio of cross-sectional area for p- and n-type TE elements has usually been optimized in previous studies by considering the material homogeneous ${ }^{(1-6,12,16,17)}$, but here the same dimensional size was applied to two kinds 
of TE element, in order to extract the other factors.

In the simulation, three-dimensional heat conduction through solid materials, temperature dependencies of the properties of the materials and the black body heat radiation in vacuum are considered. $\mathrm{Bi}_{x} \mathrm{Sb}_{2-x} \mathrm{Te}_{3}{ }^{(8)}$ properties are used to simulate the physical properties of p-type TE materials, and those of the n-type TE materials are taken from $\mathrm{Bi}_{2} \mathrm{Te}_{3-x} \mathrm{Se}_{x}{ }^{(8)}$. These TE properties are listed in Table I as a function of temperature $T^{(8)}$. The properties of electrode and insulator materials are selected as pure copper and pure alumina ${ }^{(8)}$, respectively. These materials have been commonly used by our group ${ }^{(2-9,12-14,17)}$ in previous studies. The thickness of the electrodes and the insulators are kept at $0.2 \mathrm{~mm}$ in all models. The hot source $(500 \mathrm{~K})$ is set over the top surface of the TEM, and a constant heat is supplied onto the top surface to simulate the radiated heat. The cold source (300 K) is set beneath the lower surface of the TEM to keep the cold surface of the insulator at a temperature of $300 \mathrm{~K}$. The environmental temperature of the system is set constant at $300 \mathrm{~K}$.

This work also examines the effects of the alignment of TE elements. Since the accepted model consist of 2 pairs of p- and n-type elements, four patterns of alignment can be constructed, as shown in Fig. 5: (1) small square surfaces of the elements connected to the hot surface, (2) small square surfaces of the elements connected to the cold surface, (3) small square surfaces of the n-type elements connected to the hot surface, and (4) small square surfaces of n-type elements connected to the cold surface. In order to analyze these four patterns, a model at $\theta=90^{\circ}$ and four models at $\theta=72^{\circ}$ $(\tan \theta=3), 76^{\circ}(\tan \theta=4), 81^{\circ}(\tan \theta=6)$ and $85^{\circ}(\tan \theta=12)$ are constructed, and then a total of 17 models are simulated. 


\section{Mathematical equations and procedure}

127

128

129

130

131

132

The mathematical fundamentals of TE power generation is here explained briefly $^{(2-9,12-14,17)}$. Under TE conditions, the heat conduction equation in consideration of the endothermic and exothermic Peltier effects is given by

$$
\nabla \cdot(\kappa \nabla T)+|\boldsymbol{J}| / \sigma-T \boldsymbol{J} \cdot \nabla S=0,
$$

where $\kappa, J, \sigma$ and $S$ are the thermal conductivity, current density, electric conductivity, and Seebeck coefficient, respectively. $\kappa, \sigma$ and $S$ are defined as a function of temperature $T$. The equation of the electric field in consideration of the Seebeck effect is given by

$$
\nabla \cdot(\sigma \nabla V+S \sigma \nabla T)=0
$$

where $V$ is the electric potential. These two differential equations should be simultaneously solved in three-dimensional space. The maximum power and maximum efficiency are calculated by optimizing the external load of the module. The power $P$ generated by the TEM is calculated using the equation

$$
P=V I=V^{2} / R
$$

where $V, I$, and $R$ are voltage, current, and the internal resistance of the TEM, respectively. Both $V$ and $R$ are calculated in the simulation for a given design of the module, and $V$ and $I$ are the sum of the simulated values obtained by integrating over all the meshes defined. The current $I$ is determined in order to obtain the power and the efficiency, and their maxima are obtained by optimizing the external resistance. 15-20 kinds of resistance were examined to obtain the optimum current in order to get the maximum power, $P$. The efficiency $\eta$ is then calculated using the equation

$$
\eta=P / Q_{\text {input }},
$$

where $Q_{\text {input }}$ is the heat input from the hot surface of the TEM. Note that $Q_{\text {input }}$ is divided into three parts in our model: TE power, conductive heat transmitted to the cold 
surface and heat dissipated in the surrounding environment as radiation heat. Here $Q_{\text {input }}$ is set $500 \mathrm{~mW}$, in order to avoid melting the materials by overheating.

The TEM performance was simulated using the commercial software FLUENT (ANSYS Co. Ltd., version 14.0). This software uses the finite-element method (FEM) and can solve heat transfer through solid materials and thermal fluids including heat radiation. Our original program ${ }^{(16,17)}$ was embedded in FLUENT to solve the thermoelectric and electric equations governing the Seebeck effect, Peltier effect, and Joule heat generation simultaneously. The hot surfaces were considered to be heated by radiation from the hot source at $500 \mathrm{~K}$ and all the surfaces are cooled by radiation in the environment, which is considered to be at normal temperature. The surface of the cold substrate was assumed to be cooled at constant temperature of $300 \mathrm{~K}$ by sufficiently large cold source, such as a cool water circulation system. The external conditions are different with Ref. 8, where the terminal temperatures were fixed.

The whole analysis of the performance of the TEM took a few hours to complete and the final solution after enough iterations (about 2500) showed a residual error smaller than $10^{-6}$. The convergence became slower and the iteration times larger than those achievable assuming the condition of constant temperature difference ${ }^{(8)}$. In the FEM analysis the meshes of the model of the TEM are finely divided to assure sufficient preciseness, while the air surrounding the TEM is divided coarsely. This insures both calculation accuracy and shorter calculation time. In addition, the resistance of the electric contact was not considered in the numerical simulation.

\section{Simulation of TEM performance}

\subsection{Distribution of temperature}


Fig. 6(1)-(4) show the temperature distributions on the surfaces for the four different patterns of the TEM. The TE elements are square truncated pyramids of $\theta=72^{\circ}$. For comparison, the temperature distribution of the TEM whose elements are cubic $(\theta=$ $90^{\circ}$ ) is also shown in Fig. 6(0). Fig. 6 shows that the cold insulator and cold electrodes are cooled off homogeneously regardless of the shape; the surface temperature of cold electrodes is kept at $300 \mathrm{~K}$ and it is almost the same as the terminal temperature of the TE elements because the electrode material has high thermal conductivity. It can be clearly seen from the temperature distributions that there is no heat loss caused by heat conduction between the two different materials: this is due to the exclusion of the contact thermal resistance from the calculation. As seen in Fig. 6, the upper substrate is the hottest part in all TEM models, but amongst the 4 patterns shown in the figure the highest temperature is reached by pattern 1 .

Fig. 7 shows the temperature distribution in the central part of the TE element. P-type elements in pattern $0\left(\theta=90^{\circ}\right)$ and pattern $2\left(\theta=72^{\circ}\right)$ were taken for analysis. The vertical cross-section at the center of a TE element was cut off from the three-dimensional model, and the contour map of temperature was projected onto the section. The temperature profile in pattern $0\left(\theta=90^{\circ}\right)$ is completely homogeneous and symmetric from the top surface to the bottom surface, as seen on the square projection (Fig. 7(a)), while the temperature interval in pattern $2\left(\theta=72^{\circ}\right)$ is inhomogeneous and shows a fan shaped irregular increment (Fig. 7(b)).

In the case of pattern $0\left(\theta=90^{\circ}\right)$, heat flows homogeneously without any stagnant point, as shown in Fig. 7(a). In the case of pattern $2\left(\theta<90^{\circ}\right)$, the horizontal cross-sectional area at the hot side is wider than that at the cold side. Therefore, the upper part of the TE element is well heated by radiation, or the heat remains stagnant in 
the upper part. The highest temperature, located at a corner of the upper surface of the TE element $\left(\theta=72^{\circ}\right.$ at Fig. $\left.7(2)\right)$, was $457.1 \mathrm{~K}$. However, the lower part of the TE element is cooled by the cold substrate (300 K) and the electrodes, as shown in Fig. 5. A sharp temperature drop can be seen in Fig. 7(b) at the colder side, because the lower surface area is much smaller than the upper surface area.

Fig. 8 shows the temperature distribution on the upper surfaces of the TE element terminal, the surface of which is welded with the top substrate (heat receiver). The p-type element in pattern 0 is again taken for analysis. The temperature profile on the surface of pattern 0 looks homogeneous, although a slight deviation, within $0.2 \mathrm{~K}$, can be found. The right corner of the surface shown in Fig. 8 is located close to the center of the TEM, while the left corner is close to the square edge of the top substrate. The heat homogenously irradiated onto the top substrate should flow into the TE element, and go to the TE element, the opposite terminal of which is cooled by the cold substrate. All the substrates are designed to have larger sizes than the upper surfaces of the TE elements, as shown in Fig. 4 and 5. Because of the larger amount of heat per unit area of the TE element that is accumulated onto the corners of the substrate, the heat is concentrated to the left edge of Fig. 8. This is why the left corner is about $0.25 \mathrm{~K}$ hotter than the other temperature profile at the upper surface of TE elements was a common feature of all the patterns.

All the edges of the upper square surface of the TE elements are about $0.3 \mathrm{~K}$ cooler than the central part, as shown in Fig. 8 where they appear blue. This reflects the cooling effect due to thermal radiation from the side surface of the TE elements. In 
addition, this behavior was the same for all the patterns. Although the temperature was not homogeneous (within $0.3 \mathrm{~K}$ deviation), it may be said that is almost constant in the scale of Fig. 6 . The temperature difference $(\Delta T)$ between two substrates exceeds 157.1 $\mathrm{K}$ in pattern 2 (Fig.7 (b)), if the average temperature is taken. When $\theta$ decreases to values lower than $90^{\circ}$, the temperature of the upper surface increases and $\Delta T$ becomes larger. This effect will be discussed later.

Fig. 6 also shows that the largest $\Delta T$ between two substrates could be obtained in pattern 1 , while the smaller $\Delta T$ was obtained in pattern 4 . Concerning the temperature difference in a TEM model, it is interesting that the temperature profiles of $4 \mathrm{TE}$ elements at $\theta=72^{\circ}$ mainly depends on how the elements are aligned (among the 4 possible patterns) in respect to the surfaces, showing different behavior depending whether the smaller or the larger surface touches the hot or the cold substrate. The temperature distributions of p- and n-type TE elements in pattern 3 are similar to those of pattern 4. The temperature distributions of the square truncated pyramids in contact with the hot substrates through the smaller surfaces are similar in patterns 1,3 , and 4 , as shown in Fig. 6.

\subsection{Distribution of potential and current}

Fig. 9 and 10 show the electric potential and current density distribution, respectively, in TEM models. For the potential distribution, the part shown in red is at a higher potential and that in blue is at a lower potential. The edges of electrodes and elements that are in contact with air had different values compared to their neighbors, but there was no significant influence on the analysis because this occurred only on the top skin of the surface. The total potential difference between two electrode terminals is 
a measure of the electromotive force (EMF) gained by a TEM model. Because the lowest potential is set 0 at the inlet terminal of the electrode in the simulation, the highest EMF can be obtained at the outlet terminal in pattern $2\left(\theta=72^{\circ}\right)$, and the lowest in pattern $0\left(\theta=90^{\circ}\right)$.

For the distribution of the current density shown in Fig. 10, the red part has a higher current density while the blue part has a lower current density. The current density varies inside the TE element because of the pyramidal shape and the related internal electric resistance. In particular, the corners on the side of the base of the element have a low current density. The existence of low current density area brings the dull performance because these areas do not contribute to charge transport. This result means that the internal current tends to flow preferentially in the shorter circuit such as the central part of the TE element ${ }^{(8)}$. Meng et al. proposed a slant TE element based on this concept ${ }^{(4-6)}$.

The highest current density is often marked in the shallow electrodes, as shown in pattern 2 and 4 in Fig. 10. It is noted, however, that the patterning and width of the electrode channels did not affect the performance of the individual models. This happens because the low electric resistivity of electrode material (copper) with sufficient thickness does not affect the total performance of the TEM.

Fig. 6 (0), Fig. $9(0)$ and Fig. 10 (0) show the temperature distribution, potential distribution and current density distribution, respectively, for pattern $0\left(\theta=90^{\circ}\right)$. They show the temperature difference between the substrates $(\Delta T=$ about $65 \mathrm{~K})$, potential difference $(\Delta E=45.37 \mathrm{mV})$ and current $(I=0.32 \mathrm{~A})$ between two electrode terminals under constant heat flow (500 mW). They are much smaller than those obtained for fixed temperatures of the substrates for pattern $0^{\left({ }^{(8)}\right.}$. For example, the previous study ${ }^{(8)}$ 

$\left.=90^{\circ}\right)$.

It should be noted here that all the data given in Fig. 6, 9 and 10 were obtained after optimizing the external resistance of each TEM model for maximum power, as mentioned below.

\subsection{Power of TEM}

If a TEM is connected to an external resistance, the internal current is affected by the external resistance according to the laws of serial connection in circuit theory. Therefore, power generally varies as a parabolic function of current, and there exists a maximum for the power output. The maximum power and maximum efficiency of a certain TEM model can be obtained for the same conditions of current, i.e., the same external resistance. Because Peltier heat works in addition to Joule heat, the current generated has a non-linearly relationship with the voltage. The internal resistance depends on temperature as a function of the properties of the materials; moreover, the Peltier effect also depends on temperature. In this study, we numerically simulate them both by controlling the external resistance.

Fig. 11 shows an example of the voltage, $V$, power, $P$, and efficiency, $\eta$, as a function of the current, $I$, for $\theta=85^{\circ}$ in pattern 2. As typical behavior of an electric serial circuit, the power obtainable from the TEM decreases at currents both smaller and larger than the optimal current. A small deviation in $P$ near $P_{\max }$ is due to a small uncertainty in numerical calculation and the non-linear behavior of $V$ as function of $I$.

292 The latter is due to non-linear temperature dependencies of the properties of the material 293 in TE effects. 
The evaluated maximum power $P_{\max }$ is summarized as a function of $\theta$ in Fig. 12. The

295

296

297 largest $P_{\max }$ in this work is obtained at $\theta=72^{\circ}$ for all of the alignment patterns of the TE element. It is noted that $\theta=72^{\circ}$ is the lowest angle in the studied range. A model with an angle smaller than $72^{\circ}$ was not easily constructed in the numerical mesh generation process. Overly thin cells did not give stable and reproducible results during numerical calculation. In this work, the mesh forming policy in the TEM model construction was unified and fair for all the models. It is noted that smaller cells in simulation need a larger computer capacity. The experiments with an angle smaller than $72^{\circ}$ may overcome this numerical difficulty to obtain the larger $P_{\max }$.

The electric voltage becomes larger when $\theta$ is smaller than $90^{\circ}$, as shown in Fig. 9, because the temperature difference $\Delta T$ increases. It is clear that, according to Eq. (3), the obtainable $P_{\max }$ becomes larger when the electromotive force increases. The trend of an increasing $P_{\max }$ with a reducing $\theta$, therefore, is a common feature found in all the studied alignment patterns of the TE elements (Fig. 12).

The maximum power obtainable with the assumption of constant temperature difference ${ }^{(8)}$ was compared with this work in Fig. 12. $P_{\max }$ for constant $\Delta T$ decreased as $\theta$ decreased. This trend was opposite to that found in this work (constant heat flux). For example, $1.869 \mathrm{~W}$ was lost as penetration heat in the pattern 0 , when $\Delta T=200 \mathrm{~K}$ and $\theta$ $=90^{\circ}$, and this heat decreased as $\theta$ decreased. However, in this work, we assume a constant heat flux of only $500 \mathrm{~mW}$ for all the models, and we find that the temperature difference increases due to the effect of the TE element shape as $\theta$ decreases.

\subsection{Radiation from TEM}

Fig. 12 shows that the modification of the shape of an element has a strong influence 
on $P_{\max }$ as a function of $\theta$, while the patterns of the element alignment have a relatively smaller influence on $P_{\max }$ than the contribution of $\theta$. This sensitivity to the alignment may come both from the contributions of temperature dependencies of the properties of various materials and from heat exchange among the other TE elements due to heat radiation. Here the contribution from the radiation is analyzed.

Fig. 13 shows the numerical summation of heat, $Q$, radiated from all the trapezoidal side surfaces of the TE elements. The ratio of $Q / Q_{0}$ varies from $1 \%$ to $7 \%$ depending on $\theta$, where $Q_{0}$ is the constant heat flux (500 $\mathrm{mW}$ ). Because the temperature of a TE element surface is hotter (Fig. 6) as $\theta$ decreases, it is natural that the amount of heat radiation becomes larger. It is additionally noted that the side surface area, $S$, is larger (Fig. 4) as $\theta$ decreases. For example, $S$ at $\theta=72^{\circ}$ is $18 \%$ larger than $S$ at $\theta=90^{\circ}$. Radiant heat from a wider $S$ is larger, as shown Fig. 13. Therefore, the increment of side surface area of the TE elements and the heating of these surfaces are two major reasons why we found an increased amount of radiation heat for smaller $\theta$.

At a certain $\theta$, the heat radiation in pattern 1 is smaller than in pattern 2 . The amounts of radiation heat in pattern 3 and pattern 4 are almost identical within numerical error, as seen in Fig. 13. This evidences a good symmetry of alignment of the TE elements in these two patterns. Generally, the radiated heat is not converted into electricity, and it lowers the TE performance. Therefore, the smaller $P_{\max }$ of pattern 1 compared to that of pattern 2 (Fig. 12) is partially due to the radiation loss of penetrating heat, although the amount of radiation heat is less than $7 \%$ that of penetration heat, $Q_{\text {input }}$. If we assume that the radiation plays some role in $P_{\max }$, the difference in $P_{\max }$ between patterns 3 and 4 is due to the characteristics of the materials such as electric and thermal conductivities. 

with that of shape. Moreover, the amount of radiation heat depends on the shape because of the surface area and temperature profile. The effects of different TE elements alignments to radiation are well limited as shown in Fig. 13. This short summary is valid in the mild temperature range studied in this work. When the TEM is heated to a temperature range where radiation becomes dominant, its contribution cannot be longer neglected.

\subsection{Efficiency of TEM}

Since the efficiency, $\eta$, is defined as in eq. (4), $\eta$ is proportional to $P$, and the optimum current that achieves $P_{\max }$ is identical to that that achieves also $\eta_{\max }$, as shown in Fig. 11. The angular dependence of the maximum efficiency $\eta_{\max }$ was, however, different from that found in our previous work ${ }^{(8)}$, as shown in Fig. 14. The largest $\eta_{\max }$ at constant heat flux conditions was obtained when $\theta=72^{\circ}$ in pattern 2 . In this alignment, all the small square surfaces of the element terminals are connected to the cold surface. Because the high temperature zone is extended to the cooler part as shown in Fig. 6 (2), a large amount of heat is lost as radiation from the side surfaces as shown in Fig. 13. Even so, a high temperature difference and the corresponding high potential difference increase the output power, and the highest efficiency in TE power generation is obtained in pattern 2 .

Fig. 14 also shows that the $\eta_{\max }$ at constant temperature difference were larger than those at constant heat flux conditions. The former is not so seriously dependent on angle, and the latter exceeds the former especially at the smaller angles such as $\theta=72^{\circ}$. 
shape of the TE element in consideration of the best matching between the temperature distribution of the materials and the properties of the materials in the given temperature range.

\section{Conclusion}

The combination of square truncated pyramid elements and flat plate shape was examined using numerical simulation. Taking into account the temperature dependence of the materials, the possibility of constructing a suitably shaped TEMs optimized for high efficiency was proposed. Under the constraint of constant heat flow through the TE module, the output of the TEM and the yield of input energy were numerically evaluated by solving the related differential equations. The angle $\theta$ between the edges of the square truncated pyramid was taken as an analytical parameter to describe the TE shape, and the smallest $\theta$ gave the greatest output and the higher yield. This happens because the suppression of heat transfer in the TE elements due to small cross-sectional area can give the larger temperature difference. This trend was opposite in the case of constant temperature assumption as reported in our previous study. elements.

\section{Acknowledgments}

The program used here was originally coded by Dr. Min Chen at Aalborg University, 
390 Denmark, and revised by Mr. Takeyuki Fujisaka at Hokkaido University (now at 391 Nippon Steel \& Sumitomo Metal Co., Japan), and was modified specifically for this 392 paper. This work was financially supported in part by Grant-in-Aid for Challenging 393 Exploratory Research (Japan Society for the Promotion of Science, No. 26630490), and 394 The Thermal \& Electric Energy Technology Foundation.

395 


\section{References}

397 [1] J. D’Angelo, E. D. Case, N. Matchanov, C. Wu, T. P. Hogan, J. Barnard, C. Cauchy,

T. Hendricks, and M. G. Kanatzidis, J. Electr. Mater., 40 (10), 2051-2062 (2011).

399

[2] R. O. Suzuki and D. Tanaka, J. Power Sources, 124 (1) 293-298 (2003).

400

[3] T. Kyono, R. O. Suzuki and K. Ono, IEEE Transactions on Energy Conversion, 18

401

(2) 330-334 (2003).

402

[4] X.-N. Meng, T. Fujisaka, K. O. Ito and R. O. Suzuki, Mater. Trans., 55 (8), 1219-1225 (2014).

404

[5] X.-N. Meng and R. O. Suzuki, J. Electr. Mater., 44 (6), 1469-1476 (2015).

405

[6] X.-N. Meng and R. O. Suzuki, Mater. Trans., 56 (7) 1092-1095 (2015).

406

[7] S. Oki, K.O. Ito, S. Natsui and R.O. Suzuki, J. Electr. Mater., 45, (3), 1358-1364

407 (2016).

408

[8] S. Oki and R.O. Suzuki, J. Electr. Mater., 46 (5) (2017) 2691-2696.

409

[9] R. O. Suzuki and D. Tanaka, J. Power Sources, 132 (1-2) 266-274 (2004).

410

[10] T. Kanno, A. Sasaki, K. Takahashi, A. Omote, H. Adachi and Y. Yamada, Appl. Phys. Lett., 101, 011906 (2012).

[11] D.T. Crane, J. W. LaGrandeur, F. Harris, L. E. Bell, J. Electr. Mater., 38 (7) (2009) $413 \quad$ 1375-1381.

414 [12] R. O. Suzuki and D. Tanaka, J. Power Sources, 122 (2) 201-209 (2003).

415 [13] R. O. Suzuki, K. O. Ito and S. Oki, J. Electr. Mater., 45 (3) 1827-1835 (2016).

416 [14] R. O. Suzuki, T. Fujisaka, K. O. Ito, X. Meng and H.-T. Sui, J. Electr. Mater., 44

(1) (2015) 348-355.

418 [15] T. Kuroki, K. Kabeya, K. Makino, T. Kajihara, H. Kaibe, H. Hachiuma, H. 419 Matsuno, A. Fujibayashi, J. Electr. Mater., 43 (6) (2014) 2405-2410. 
420 [16] M. Chen, L.A. Rosendahl, and T. Condra, Inter. J. Heat Mass Transf., 54, 345-355 421 (2011).

422 [17] T. Fujisaka, H. Sui, and R. O. Suzuki, J. Electr. Mater., 42, (7), 1688-1696 (2013). 423 


\section{Table caption}

425 Table I Thermophysical properties of two TE materials ${ }^{(8)}$

426

\section{Figure captions}

428

429

430

431

432

433

434

435

436

437

438

439

440

Fig. 1 Thermoelectric element with square truncated pyramid shape.

Fig. 2 Concept of a module with TE elements of square truncated pyramid shape.

Fig. 3 Dimensions of TE elements and the other parts (in unit of mm) ${ }^{(8)}$.

Fig. 4 Angular dependency of dimensions of the studied element. $V$ and S are volume (set constant as $1 \mathrm{~mm}^{3}$ ) and surface area of an element, respectively.

Fig. 5 Four patterns of alignments (1)-(4) at $\theta=72^{\circ}$, and an alignment (0) at $\theta=90^{\circ}$ for reference ${ }^{(8)}$.

Fig. 6 Temperature distribution in the TEM. (1)-(4) Four patterns of alignments at $\theta=$ 72 degree. (0) the distribution at $\theta=90^{\circ}$ for reference.

Fig. 7 Contour map of temperature at the central cross-section of a TE element;

(a) $\theta=90^{\circ}$ (pattern 0) and (b) $\theta=72^{\circ}$ (pattern 2).

Fig. 8 Contour map of temperature at the top surface of the TE element located at right-end corner in Fig. 5 (0); $\theta=90^{\circ}$ (pattern 0).

Fig. 9 Electric potential distribution in the TEM. (1)-(4) Four patterns of alignments at $\theta=72^{\circ}$. (0) the distribution at $\theta=90^{\circ}$ for reference.

Fig. 10 Distribution of current density in the TEM. (1)-(4) Four patterns of alignments at $\theta=72^{\circ}$. (0) the distribution at $\theta=90^{\circ}$ for reference.

Fig. 11 Relationship among current, voltage, power and efficiency in pattern 2 at $\theta=$ $85^{\circ}$ 
447 Fig. 12 Maximum power generated by TEM as a function of angle. Broken line:

448 maximum power in case surface temperature is fixed $\left(\Delta T=200 \mathrm{~K}^{(8)}\right)$.

449 Fig.13 Radiated heat from TE elements surface.

450 Fig. 14 Maximum efficiency generated by TEM as a function of angle.

451 
Electric conductivity, $\sigma \times 10^{2} / \mathrm{S} \mathrm{cm}^{-1}$

$$
\sigma=-473.1+0.86507 T-\mathrm{e}^{16.637-1.6942 \ln (T)}
$$

Seebeck coefficient, $S / \mu V K^{-1}$

$S=-188.2+2.2411 T-3.0075 \times 10^{-3} T^{2}+2.4914 \times 10^{-7} T^{3}$

Thermal conductivity, $\kappa / \mathrm{W} \mathbf{m}^{-1} \mathrm{~K}^{-1}$

$\kappa=-1.8067+5.7529 \times 10^{-3} T-64.639 / T+1.3395 \times 10^{5} / T^{2}$

Material:n-type $\mathrm{Bi}_{2} \mathrm{Te}_{3-x} \mathrm{Se}_{x}$

Electric conductivity, $\sigma \times 10^{2} / \mathrm{S} \mathrm{cm}^{-1}$

$\sigma=-2139.4+2.5778 T+\mathrm{e}^{12.765-0.89092 \ln (T)}$

$300-560$

Seebeck coefficient, $S / \mu V K^{-1}$

$$
S=443.49-4.5121 T+9.4424 \times 10^{-3} T^{2}-5.8362 \times 10^{-6} T^{3}
$$

Thermal conductivity, $\kappa / \mathbf{W} \mathbf{m}^{-1} \mathbf{K}^{-1}$

$$
\kappa=-4.6205+833.7 / T+23536 / T^{2}
$$




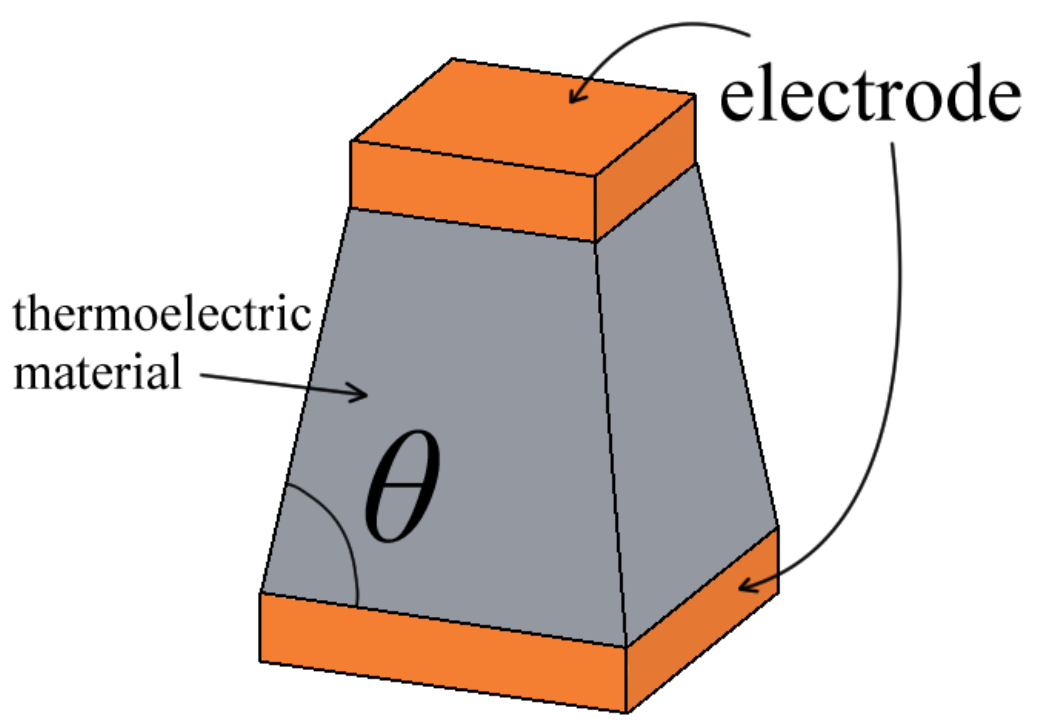

459

Fig. 1 Thermoelectric element with square truncated pyramid shape.

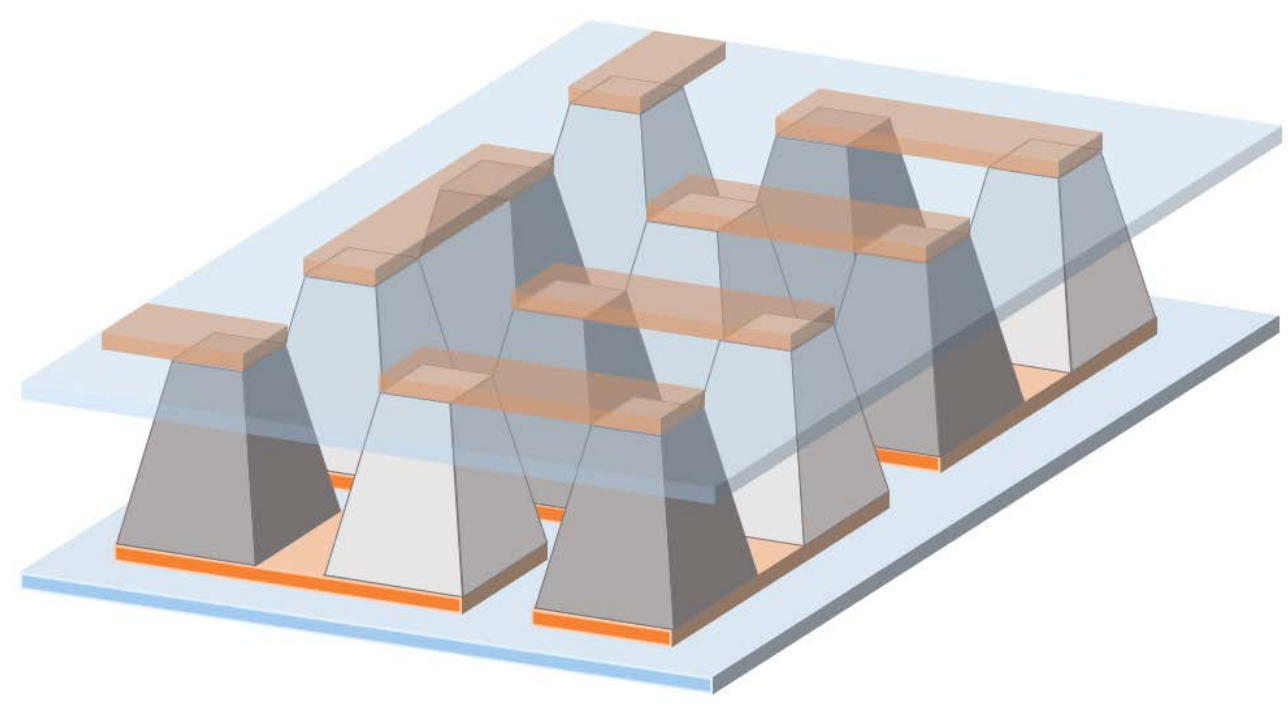

463

Fig. 2 Concept of a module with TE elements of square truncated pyramid shape. 


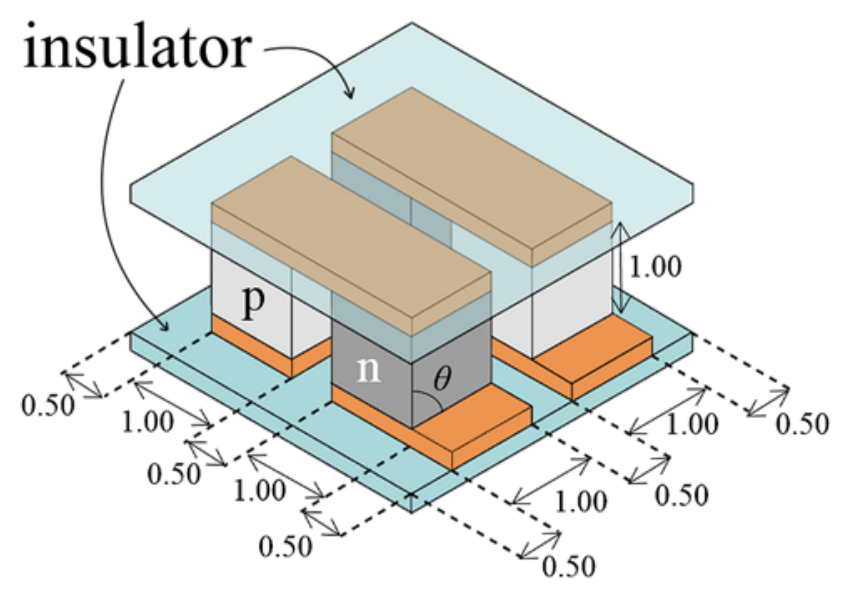

Fig. 3 Dimensions of TE elements and the other parts (in unit of mm) ${ }^{(8)}$.

468

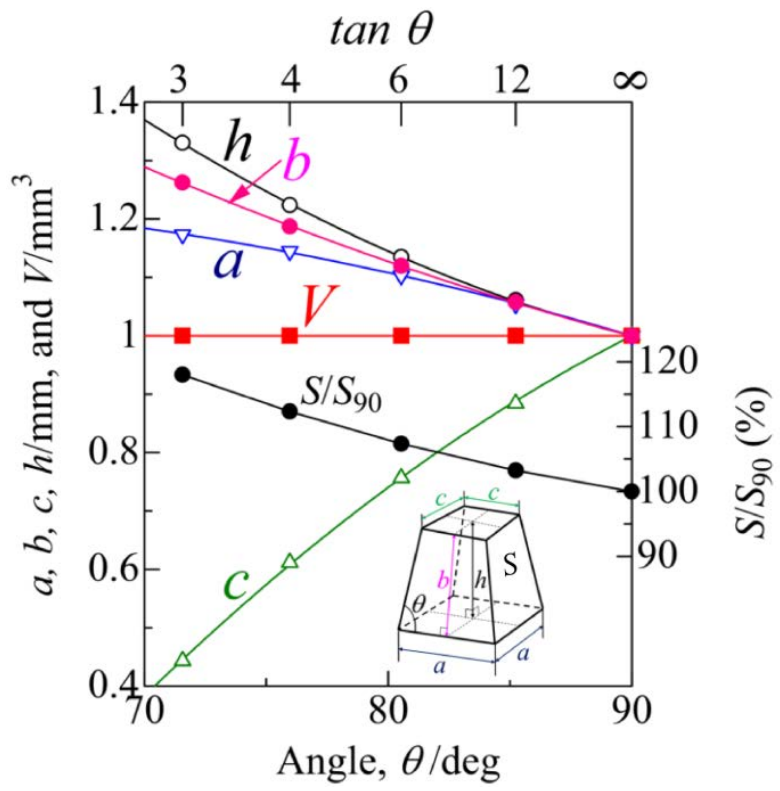

470

Fig. 4 Angular dependency of dimensions of the studied element. $V$ and S are volume (set constant as $1 \mathrm{~mm}^{3}$ ) and surface area of an element, respectively. 


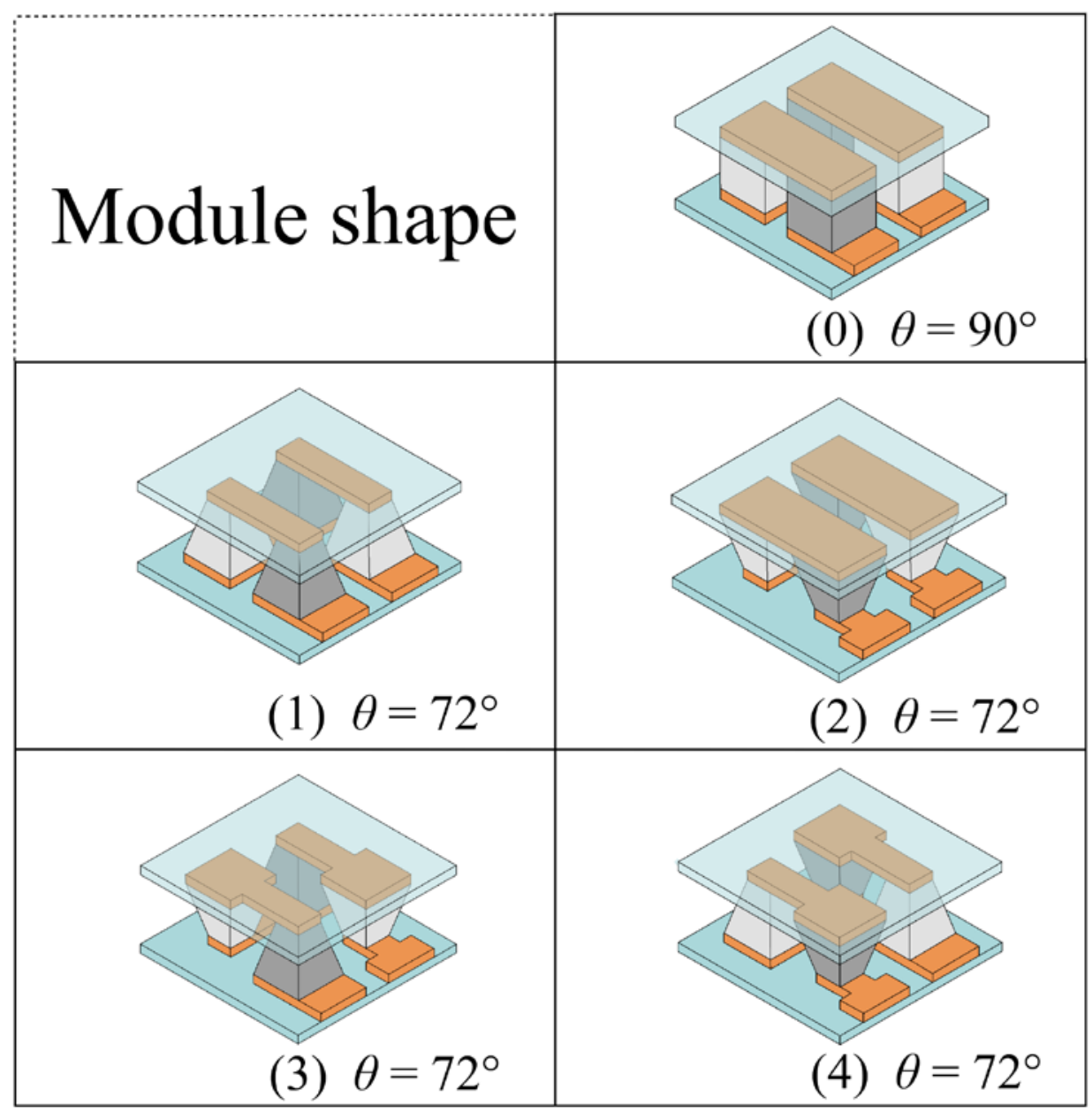

Fig. 5 Four patterns of alignments (1)-(4) at $\theta=72^{\circ}$, and an alignment (0) at $\theta=90^{\circ}$ for reference ${ }^{(8)}$. 


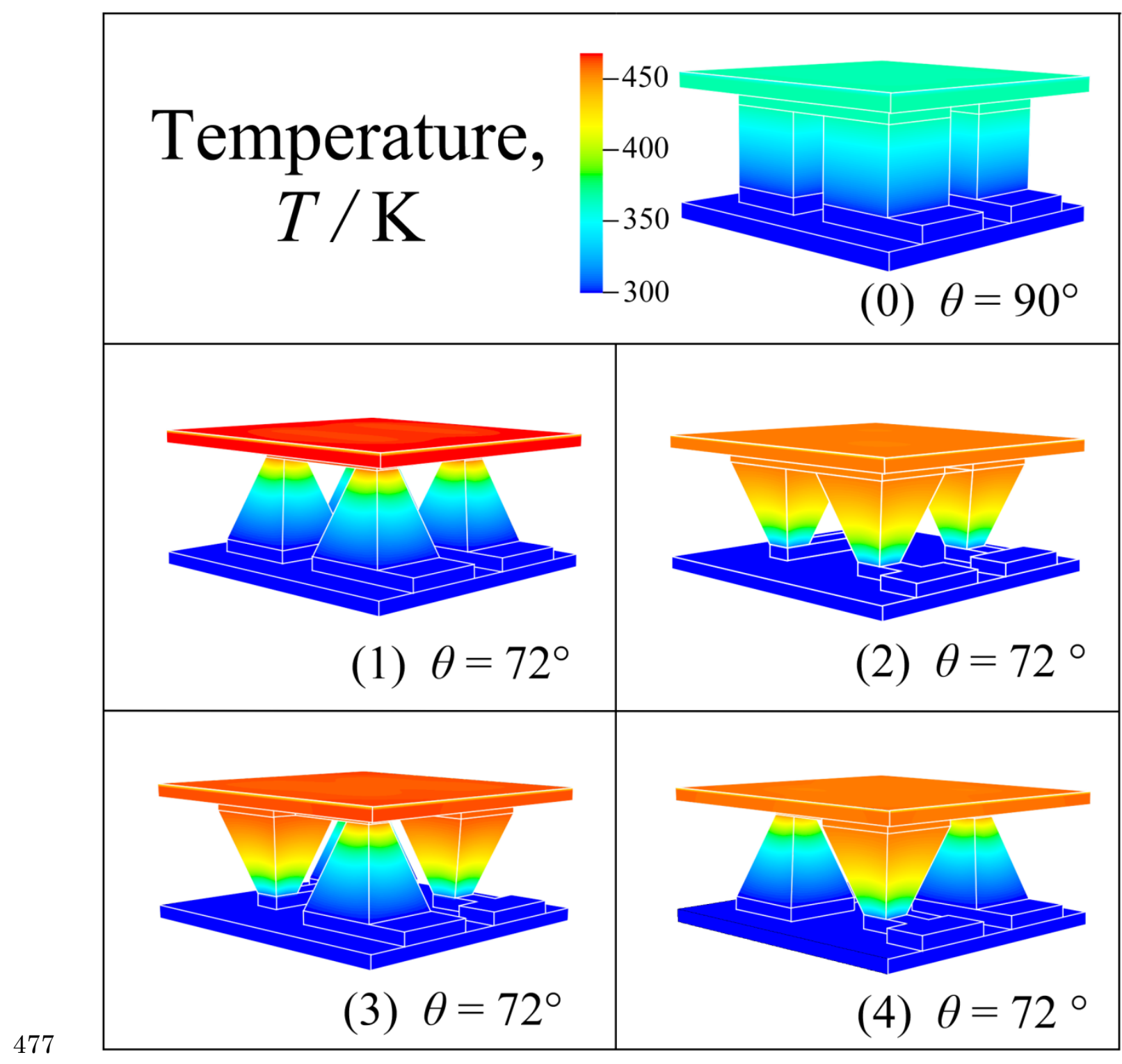

478

Fig. 6 Temperature distribution in the TEM.

(1)-(4) Four patterns of alignments at $\theta=72^{\circ}$.

481

(0) the distribution at $\theta=90^{\circ}$ for reference. 


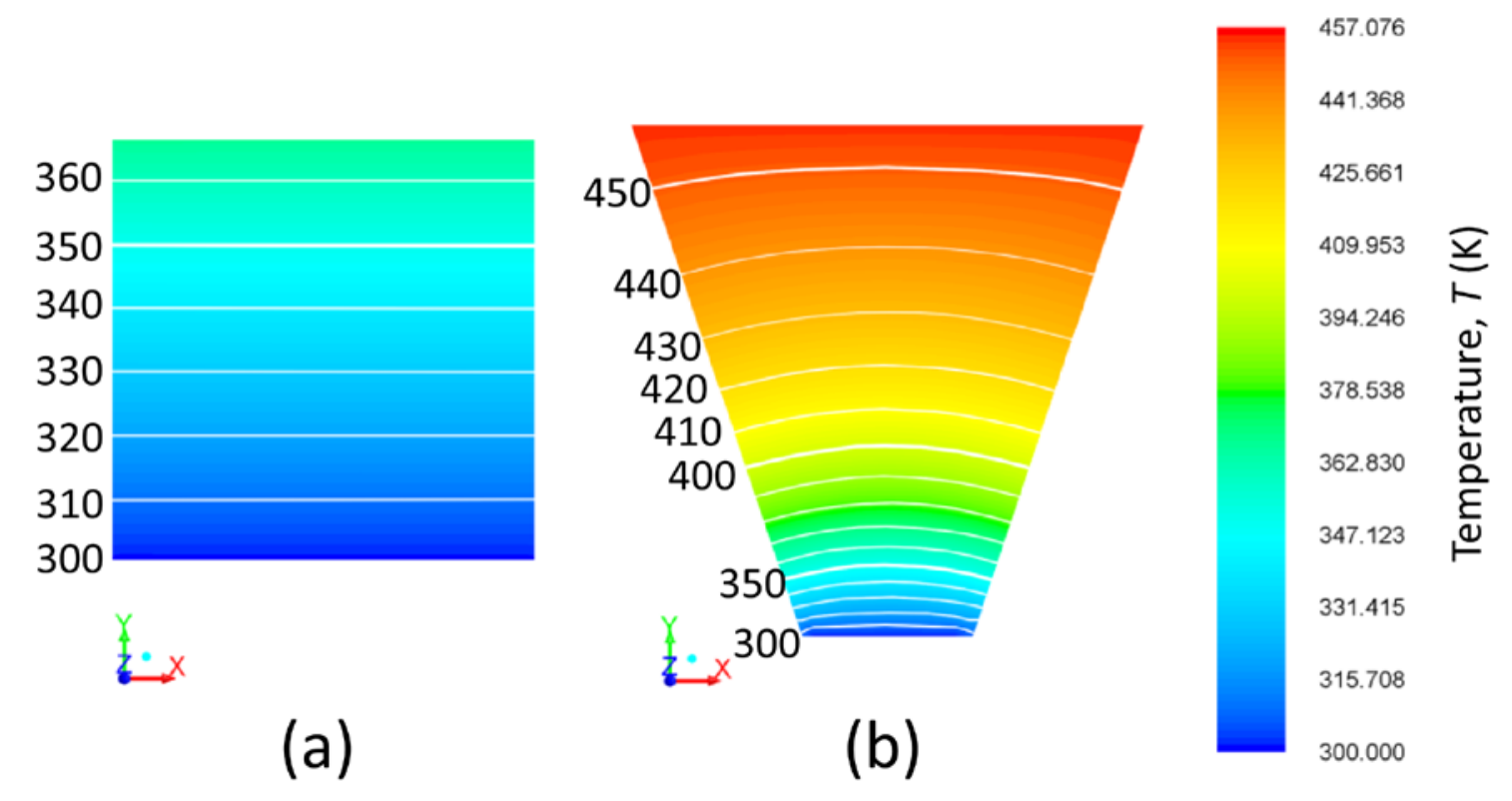

484

Fig. 7 Contour map of temperature at the central cross-section of a TE element;

(a) $\theta=90^{\circ}$ (pattern 0) and (b) $\theta=72^{\circ}$ (pattern 2).

487

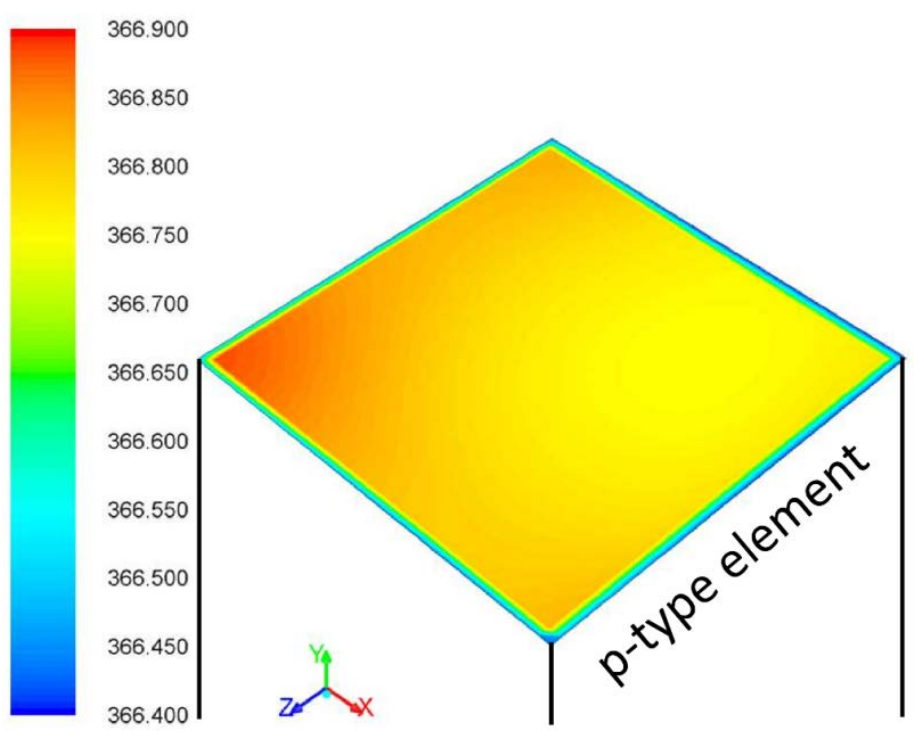

490 Fig. 8 Contour map of temperature at the top surface of the TE element located at 491 left-end corner in Fig. 5 (0); $\theta=90^{\circ}$ (pattern 0). 


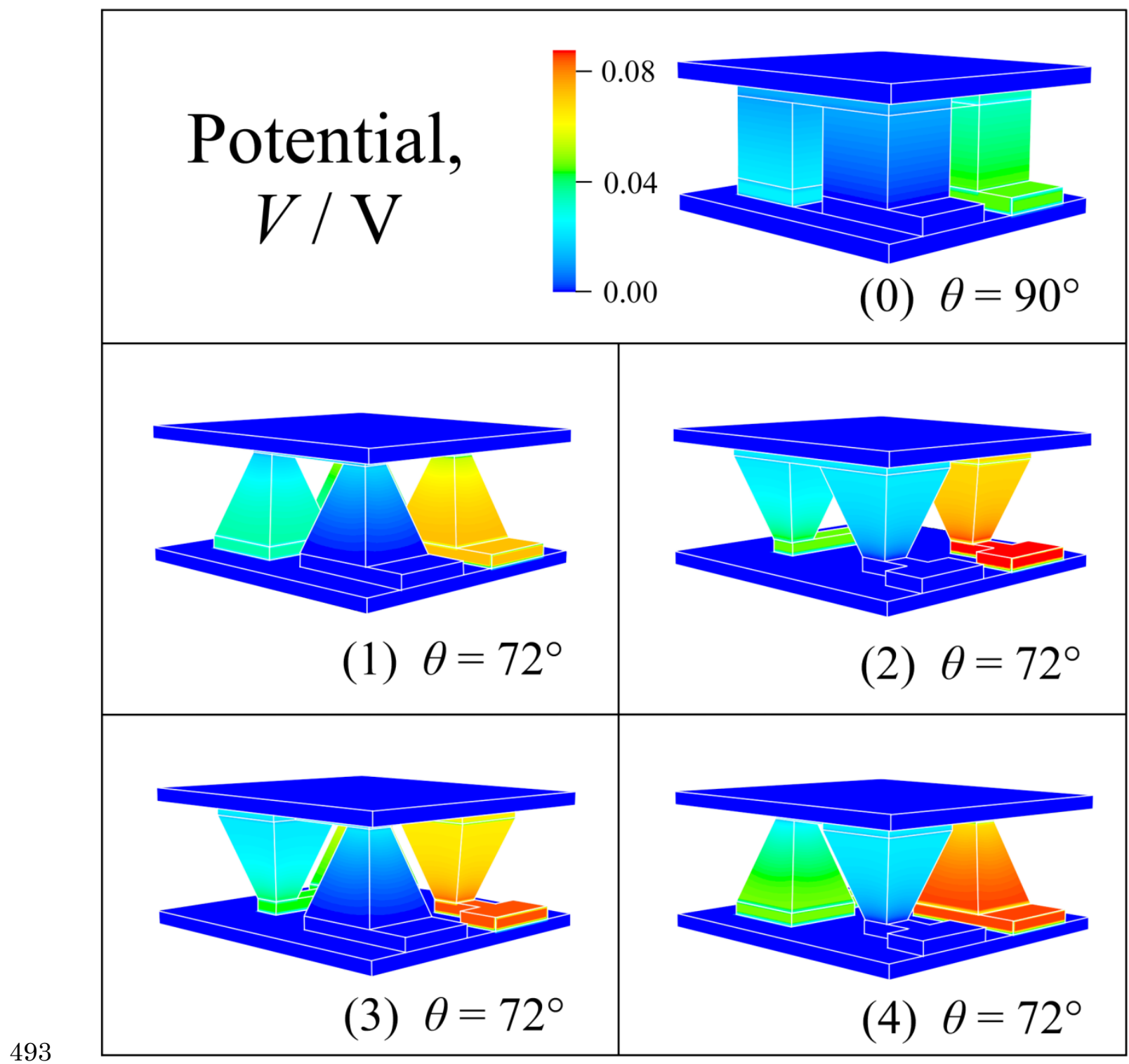

Fig. 9 Electric potential distribution in the TEM.

(1)-(4) Four patterns of alignments at $\theta=72^{\circ}$.

498

(0) the distribution at $\theta=90^{\circ}$ for reference. 


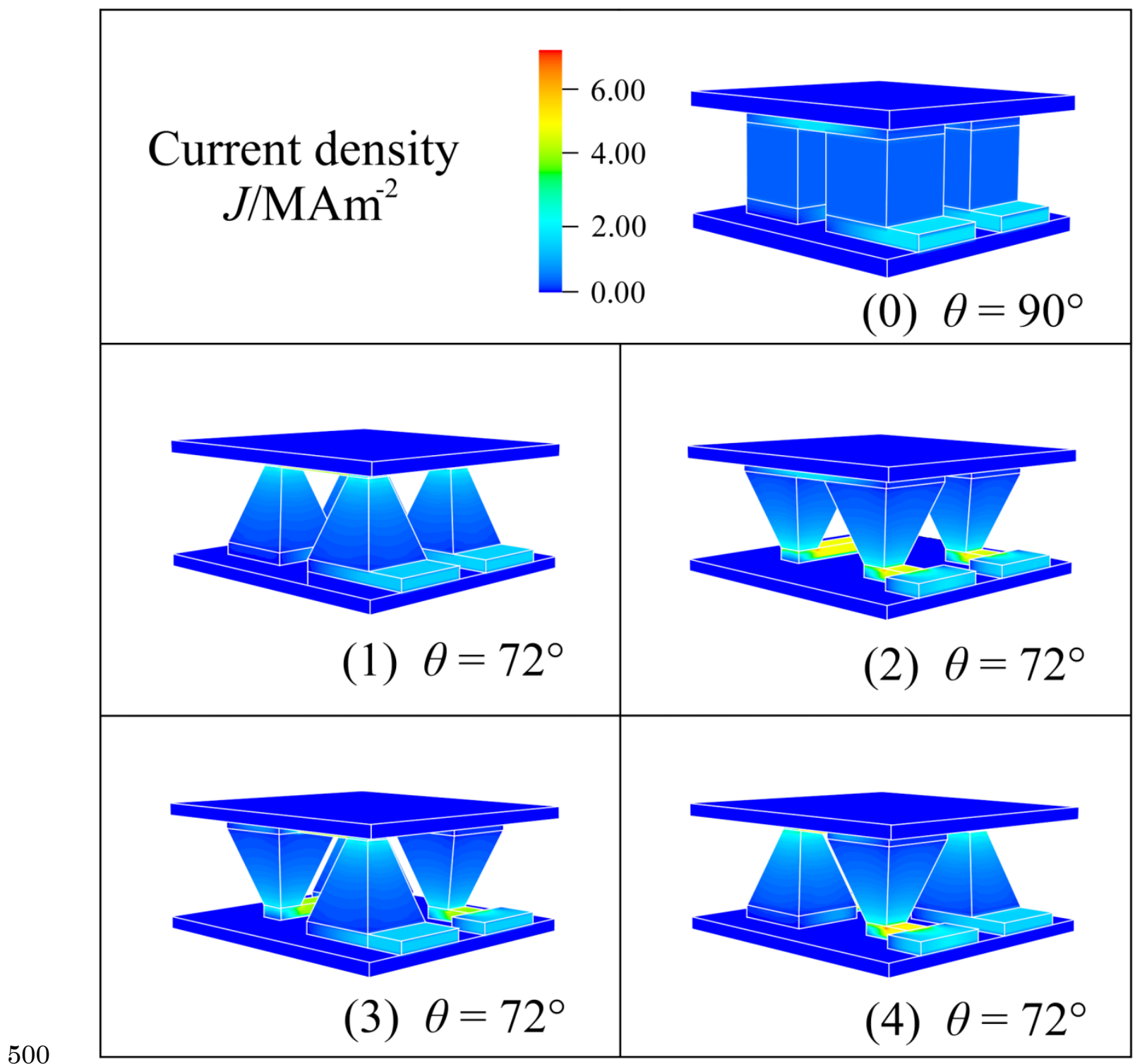

Fig. 10 Distribution of current density in the TEM.

(1)-(4) Four patterns of alignments at $\theta=72^{\circ}$.

505

(0) the distribution at $\theta=90^{\circ}$ for reference.

506 


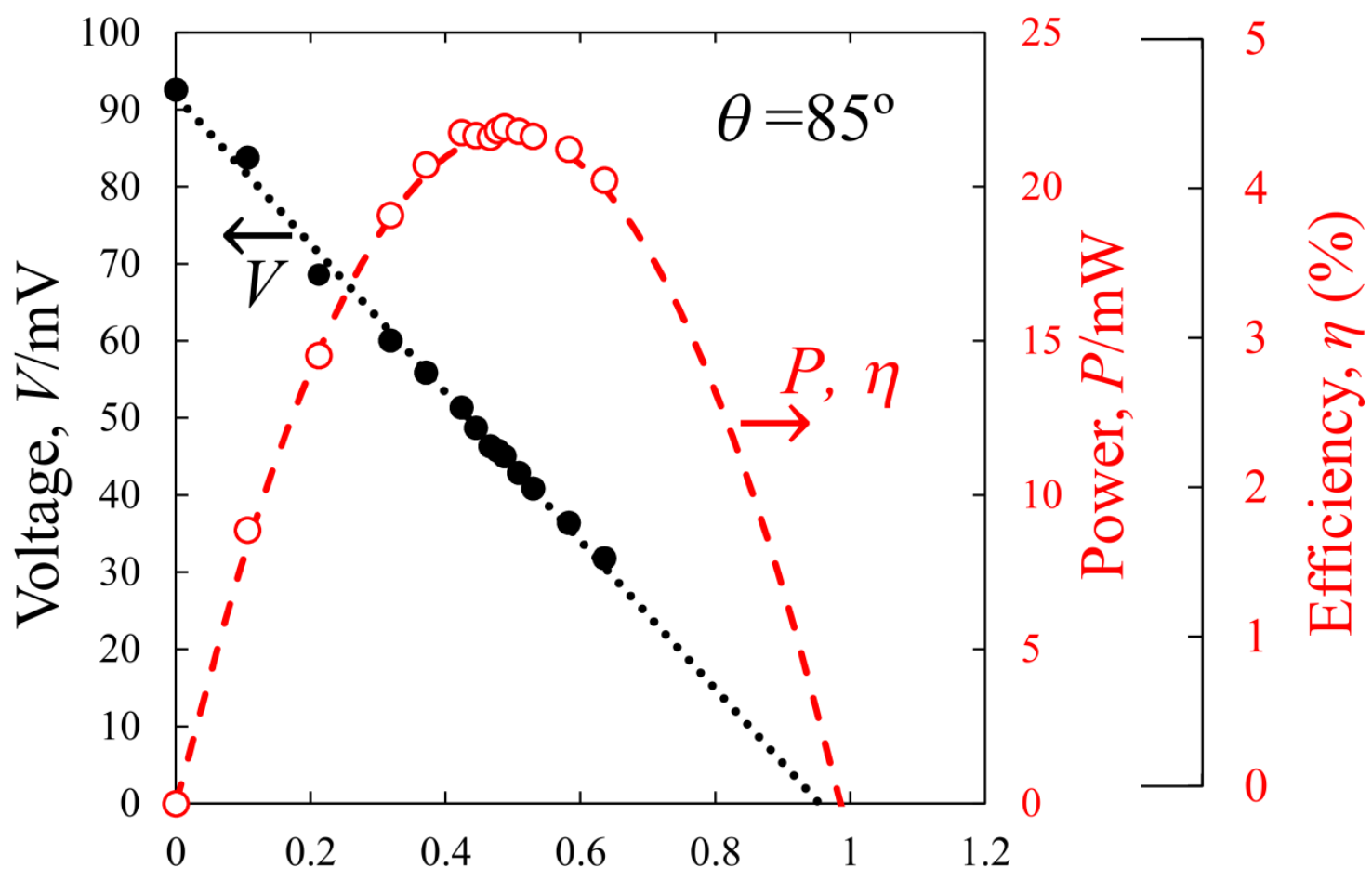

507

Current,I/A

508

509 Fig. 11 Relationship among current, voltage, power and efficiency in pattern 2 at $\theta=$ $51085^{\circ}$.

511 


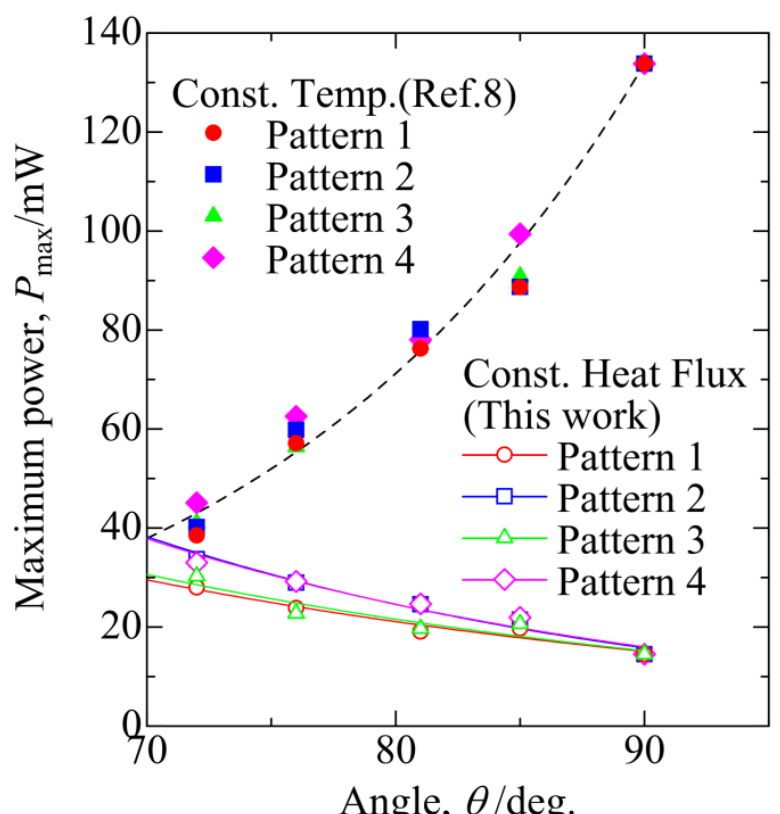

Angle, $\theta /$ deg.

513

514 Fig. 12 Maximum power generated by TEM as a function of angle. Broken line:

515 maximum power in case surface temperature is fixed $\left(\Delta T=200 \mathrm{~K}^{(8)}\right)$. 


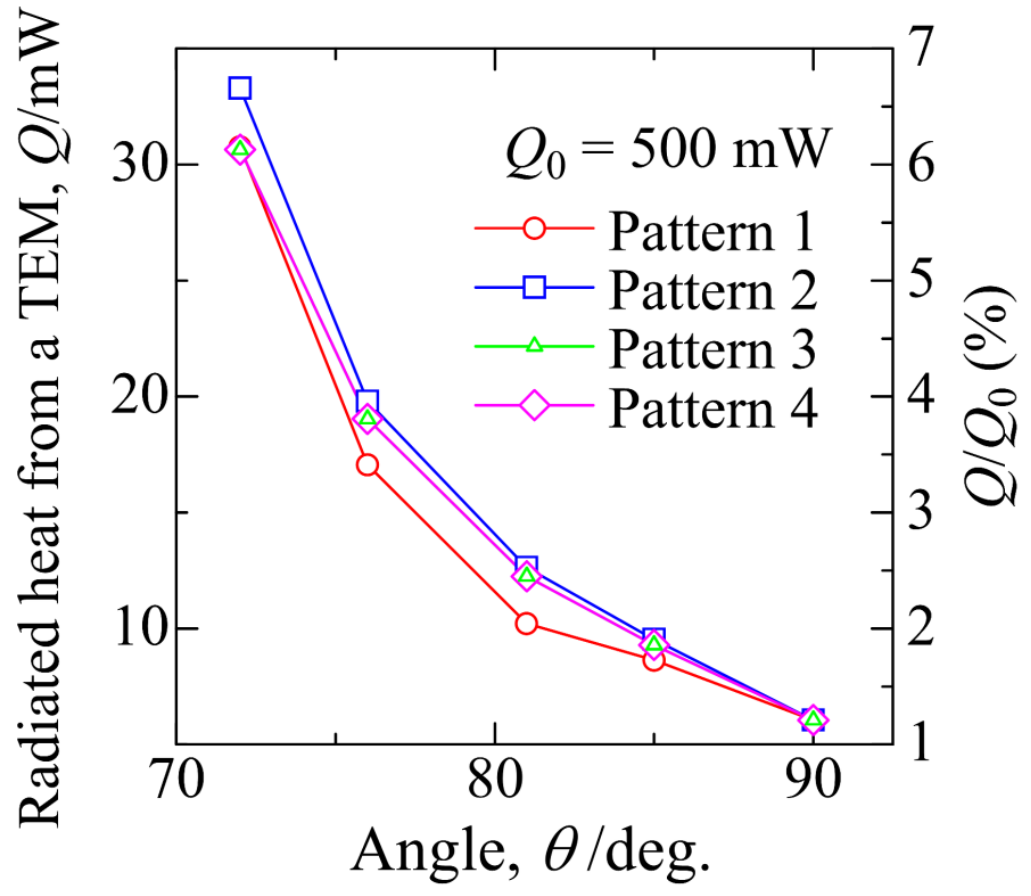

518

519

Fig.13 Radiated heat from TE elements surface.

520 


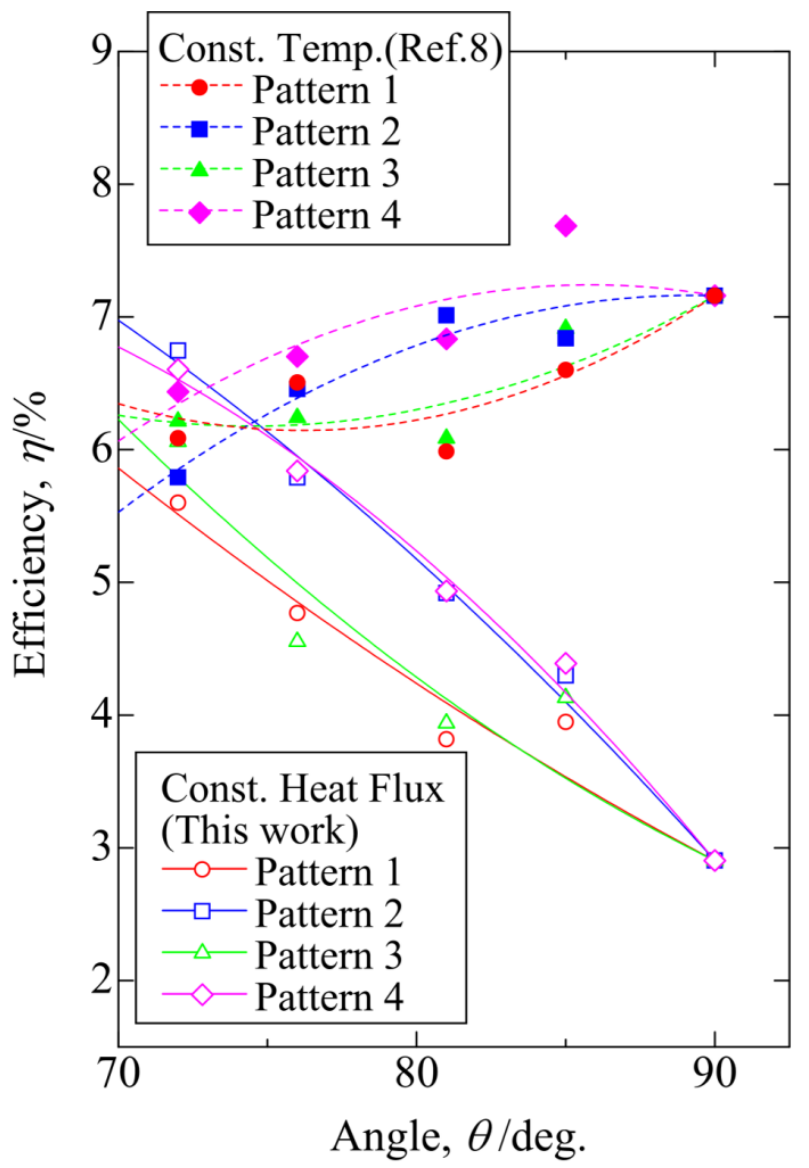

